\title{
Plasticity and modular control of locomotor patterns in neurological disorders with motor deficits
}

\section{Y. P. Ivanenko ${ }^{*}$, G. Cappellini ${ }^{1,2}$, I. A. Solopova ${ }^{3}$, A. A. Grishin ${ }^{3}$, M. J. MacLellan ${ }^{1}$, R. E. Poppele ${ }^{4}$ and F. Lacquaniti ${ }^{1,2,5}$}

1 Laboratory of Neuromotor Physiology, Santa Lucia Foundation, Rome, Italy

${ }^{2}$ Centre of Space Bio-Medicine, University of Rome Tor Vergata, Rome, Italy

${ }^{3}$ Laboratory of Neurobiology of Motor Control, Institute for Information Transmission Problems, Russian Academy of Sciences, Moscow, Russia

${ }^{4}$ Department of Neuroscience, University of Minnesota, Minneapolis, MN, USA

${ }^{5}$ Department of Neuroscience, University of Rome Tor Vergata, Rome, Italy

\section{Edited by:}

Martin Giese, University Clinic

Tuebingen/Hertie Institute, Germany

Reviewed by:

Maurizio Mattia, Istituto Superiore di

Sanità, Italy

Walter Maetzler, University of

Tuebingen, Germany

${ }^{*}$ Correspondence:

Y. P. Ivanenko, Laboratory of

Neuromotor Physiology, Scientific

Institute Foundation Santa Lucia,

306 via Ardeatina, 00179 Rome, Italy

e-mail: y.ivanenko@hsantalucia.it
Human locomotor movements exhibit considerable variability and are highly complex in terms of both neural activation and biomechanical output. The building blocks with which the central nervous system constructs these motor patterns can be preserved in patients with various sensory-motor disorders. In particular, several studies highlighted a modular burst-like organization of the muscle activity. Here we review and discuss this issue with a particular emphasis on the various examples of adaptation of locomotor patterns in patients (with large fiber neuropathy, amputees, stroke and spinal cord injury). The results highlight plasticity and different solutions to reorganize muscle patterns in both peripheral and central nervous system lesions. The findings are discussed in a general context of compensatory gait mechanisms, spatiotemporal architecture and modularity of the locomotor program.

Keywords: compensation, plantarflexor weakness, spinal cord injury, stroke, EMG activity, modularity, locomotor pattern generation

\section{INTRODUCTION}

Investigating locomotor responses after neurological lesions is fundamental to the development of improved rehabilitation strategies and to explore the mechanisms involved in improving locomotor function. The problem of motor neurorehabilitation is significant and complex. Numerous studies have shown that motor activity after brain damage plays an essential role in anatomo-physiological reorganization, which may occur in the areas adjacent to the damage (Cao et al., 1998; Nelles et al., 1999). Nevertheless, the building blocks with which the central nervous system constructs the motor patterns can be preserved in patients with neurological disorders. In particular, several studies highlighted a modular burst-like organization of muscle activity.

While biomechanical and neural aspects of human locomotion have been documented in many studies both in normal and pathological gait, the architecture of neural circuits and the nature of descending neural signals that are involved in locomotor control remain elusive in humans. To date, little work has been completed on characterizing the neural substrates for modularity in both healthy individuals and in neurological patients with different sensory-motor disorders. A number of studies explored the bases of central motor programming by decomposing muscle activation patterns as a means to look backward from the periphery to the CNS (Davis and Vaughan, 1993; Prentice et al., 1998; d'Avella and Bizzi, 2005; Ivanenko et al., 2006; Giszter et al., 2007; Tresch and Jarc, 2009; Chvatal and Ting, 2012; Bizzi and Cheung, 2013; Lacquaniti et al., 2013). While different studies use different decomposition techniques, the common message is the emphasis on modular architecture of the motor output. Furthermore, these computational techniques often converge to a similar solution
(Ivanenko et al., 2005; Tresch et al., 2006). The data and concepts discussed here offer a new approach to characterizing the mechanisms underlying control of human locomotion that may potentially benefit the study of pathological gait and the ability of current therapeutic exercises to improve patient outcomes.

In patients, the mechanisms involved in locomotor improvements may rely on the inherent spatiotemporal organization of neural circuitry and its adaptability. The question arises as to whether the rhythmic patterning elements are invariant when muscle activation patterns can be compromised by spinal cord lesions, brain damage and other motor disturbances. Compensatory strategies for plantarflexor weakness or after distal limb segment amputation also represent important examples of locomotor adaptations. Several recent studies provide some clues on this topic. We will consider and discuss these examples in the following sections.

\section{LOCOMOTOR PATTERNS IN HEALTHY SUBJECTS}

Muscle activity during normal locomotion has both invariant and variant features. In each step, the control system needs to compensate for body weight, provide forward and lateral stability and maintain forward progression. The coordination of the musculoskeletal system with non-linear properties and multiple degrees of freedom is complex and requires activity of tens of leg muscles. Major muscle activity during walking tends to be organized in bursts at specific moments of the gait cycle (Figure 1A) to perform specific functions dictated by the biomechanics of bipedal walking (Winter, 1989; Zajac et al., 2003; Lacquaniti et al., 2012). For instance, in early stance hip and knee extensors contribute to weight acceptance at heel contact (Figure 1B). Ankle 


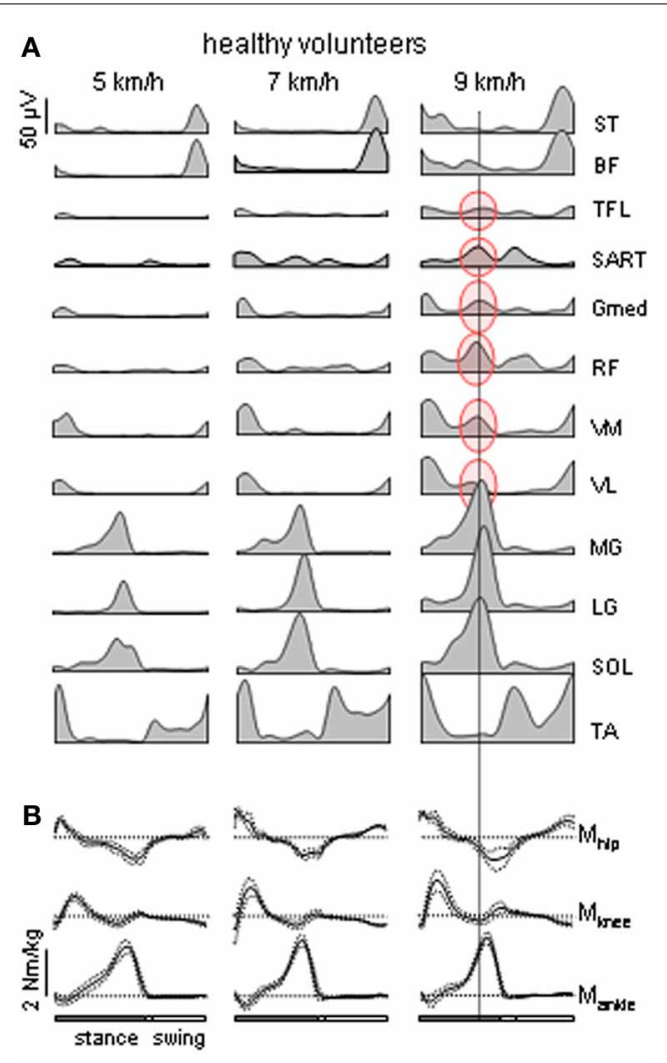

FIGURE 1 | Motor patterns in healthy volunteers. (A) Ensemble-averaged EMGs ( $n=8$ subjects) recorded from 10 ipsilateral leg muscles during walking on a treadmill at 5, 7, and $9 \mathrm{~km} / \mathrm{h}$. At $9 \mathrm{~km} / \mathrm{h}$, there is an "atypical" burst of activity in several thigh muscles that is synchronous with the peak activity in the calf muscles [the data are illustrated from Ivanenko et al. (2008)]. ST, semitendinosus; BF, biceps femoris; TFL, tensor fascia latae; SART, Sartorius; Gmed, RF, rectus femoris; VM, vastus medialis; VL, vastus lateralis; MG, gastrocnemius medialis; LG, gastrocnemius lateralis; $S O L$, soleus; TA, tibialis anterior. (B) ensemble-averaged $( \pm S D)$ ankle, knee and hip moments of force (normalized to the subject's weight) of the right leg during overground walking at about the same speeds (as in panel $\mathbf{A}$ ) in one representative healthy subject.

plantar flexors provide body support and forward propulsion in late stance while ankle dorsiflexors and hip flexors contribute to foot lift-off in early- to mid-swing. Simultaneously, erector spinae muscles activate at this time to stabilize the trunk. In late swing, hamstrings decelerate the leg in preparation for heel contact and then stabilize the pelvis. Throughout the entire step cycle, adductor muscles contribute to the control of medio-lateral accelerations of the center of body mass. However, it is worth noting that most leg muscles that are involved in the control of forward progression in the sagittal plane have a noticeable lateral component of force production and thus are also involved in the control of motion in non-sagittal planes. Even though there is a relationship between the neural and biomechanical control of the gait cycle, evidentially the system is much more complex due to the dynamic coupling of multiple body segments (e.g., Zajac et al., 2003).
Whereas this "invariant" picture of muscle activation (Figure 1A) has been documented in healthy subjects, there are also variant features of muscle activity depending on the context and differences between individuals. If one aims at reactivating the "normal" motor patterns in patients and extrapolating them to any walking condition, this may not be of benefit to the patient due to the specific pathology or individual differences that occur in pathological as well as healthy subjects. Muscle activity in healthy subjects may show very non-linear changes in both amplitude and temporal envelope, e.g., with changing the speed or body weight support even while kinematics patterns remain similar (Ivanenko et al., 2002; Lacquaniti et al., 2002). For instance, the amplitude of EMG activity of "anatomical" synergists may diverge remarkably in these conditions: lateral and medial gastrocnemius muscles at different walking speeds (Huang and Ferris, 2012), soleus and gastrocnemius muscles at different levels of limb loading (McGowan et al., 2010). With body weight unloading (Ivanenko et al., 2002), most muscles (e.g., gluteus maximus and distal leg extensors) decrease their activity, while other muscles demonstrate a "paradoxical" increment of activation (e.g., quadriceps) or considerable changes in the activation waveforms (hamstring muscles). In addition, muscle activity patterns are shaped by the direction of progression (e.g., forward vs. backward, Grasso et al., 1998, or walking along a curved path, Courtine et al., 2006). In particular, such studies suggest that a comparison of normal and pathological gait should be preferably performed in the same stepping conditions.

There is also notable inter-individual variability in muscle activity during walking (Winter and Yack, 1987). The most variable patterns are observed in the proximal and bi-articular muscles especially at lower walking speeds. For example, quadriceps activity is virtually silent in some subjects at low speeds $(<4 \mathrm{~km} / \mathrm{h}$ ), whereas it is still present in others (Ivanenko et al., 2002). Finally, notable systematic changes in the EMG activity during walking occur with age, e.g., co-contraction of leg muscles in infants (Forssberg, 1985; Teulier et al., 2012; Ivanenko et al., 2013b) and widening of EMG patterns in elderly (Monaco et al., 2010).

Nevertheless, a number of recent studies using statistical analyses of EMG suggest that the nervous system may adopt a relatively simple control strategy (e.g., Ivanenko et al., 2005; Cappellini et al., 2006; Clark et al., 2010; McGowan et al., 2010; Monaco et al., 2010). Using pattern recognition mathematics, both the stereotypical activation patterns and across-stride variability of these patterns can be accounted for by combining and scaling a small set of basic activation components. Such tendency for a few basic patterns to account for about $90 \%$ of variance indicates that leg muscles tend to group their activity in order to perform specific biomechanical functions during gait.

While intuitively one would expect some changes in the EMG activity in patients, the present question is whether the basic modular patterns or the functional grouping of muscles are conserved in pathological participants. Below we summarize various examples of motor patterns in patients with both peripheral and central lesions. The main focus is placed on the studies that analyzed multi-muscle EMG patterns. 


\section{LOCOMOTOR PATTERNS IN PERIPHERAL LESIONS}

Compensatory strategies for plantarflexor weakness or after below-knee amputation represent an important example of gait adaptation. The human bipedal gait and heel-to-toe rolling pattern are unique (Bramble and Lieberman, 2004) and require a specific inter-segmental coordination (Lacquaniti et al., 2002), balance control and walking experience for acquisition of plantigrade gait at the beginning of independent walking (Forssberg, 1985; Ivanenko et al., 2007). Plantarflexor muscles are an important muscle group that regulates the gait speed, compensates for body weight and provides the vertical and horizontal (anteriorposterior shear) forces during the push-off phase. Weakness of the plantarflexors is considered as one of the limiting factors that prevents humans from walking at faster speeds (Nadeau et al., 1999; Brunner and Romkes, 2008).

In addition to the development of extensor forces in the distal antigravity muscles, there is an important sensory feedback from these muscles and from numerous foot receptors. Peripheral neuropathy and aging may result in muscle weakness and substantial impairments of sensory feedback and balance control (Nardone et al., 2000, 2006; Nardone and Schieppati, 2004; Mazzaro et al., 2005). For instance in older adults, ankle plantarflexor work remains relatively constant at increasing speeds, in contrast to the systematic increase in ankle work output with walking speed in young adults (Winter et al., 1990; Judge et al., 1996).

\section{PATIENTS WITH LARGE FIBER PERIPHERAL NEUROPATHY}

Weakness of distal extensors in patients with large-fiber neuropathy can be observed after acute nerve compression in the sciatic notch associated with a reduced level of motor and sensory function. After sciatic nerve compression there may be a loss of reflexes, movement skills, sensation in the affected area, and atrophy of the affected muscles can occur (Hagiwara et al., 2003). Sciatica commonly refers to pain that radiates along the sciatic nerve and is typically felt in the back of the leg and possibly to the foot, and is one of the most common forms of pain caused by compression of the spinal nerves.

We analyzed adaptations of gait patterns at different walking speeds in four patients with a unilateral large-fiber neuropathy of $\mathrm{S} 1$ innervation resulting from acute nerve compression in the sciatic notch. Plantarflexor weakness on the affected side was evidenced by subjective difficulty to lift and support the body weight on the forefoot region (forefootstanding) by plantarflexing the ankle joint during standing. Reflexes and sensory thresholds were all normal in the contralateral leg. Figure 2 illustrates EMG patterns in these patients during walking and slow and fast speeds. After acute nerve compression in the sciatic notch, the patients walked somewhat slower (even their self-selected fast speed was $<5 \mathrm{~km} / \mathrm{h}$ ) than healthy individuals. The EMG patterns differed from healthy individuals walking at the same speeds (Figure 1A) and were variable between the patients as well as between left and right leg muscle activities (Figure 2). A part of these differences may originate from slightly different EMG electrode placements and/or skin impedance. Nevertheless, we found an interesting cooperation of distal and proximal extensors (Figure 2, marked in red) and discuss its general functional significance for bipedal gait adaptations (Dickey and Winter, 1992; Beres-Jones and Harkema, 2004; Nene et al., 2004; Ivanenko et al., 2008, 2013a).

The "atypical" burst of activity in the proximal leg muscle was more prominent in patient 1 (Figure 2) on the affected side, while it could be observed also in other patients on the contralateral side. To understand better it's link to the kinematics and kinetics of gait, we recorded patient 1 again during overgound (Figure 3B) and treadmill walking (Figures 3A,C). The second time ( 1 year later, session 2 ) the patient had recovered all reflexes and reduced sensation was limited to the lateral, plantar surface of the foot. The patient was now able to fully support his body weight on the left leg during "forefoot standing," although some weakness still remained. The most prominent decrements in angular oscillations and angular velocities were observed in

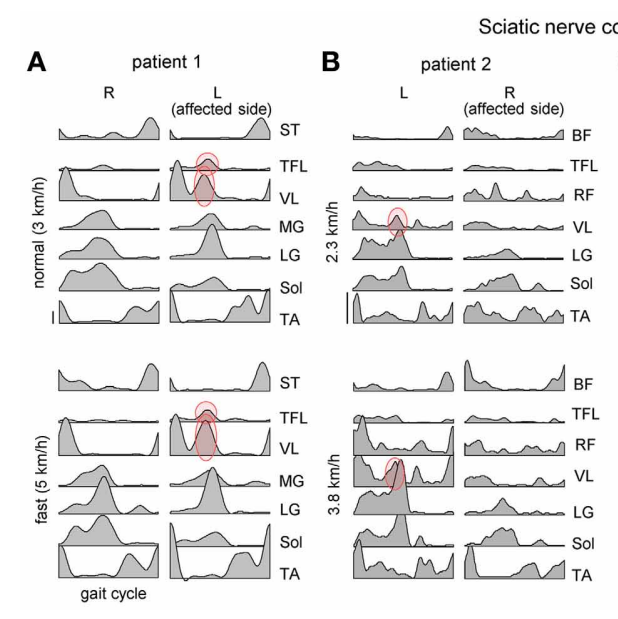

FIGURE 2 | Ensemble-averaged bilateral EMG activity of leg muscles during overground walking at slow and fast speeds in four patients (panels A-D) with unilateral sciatic nerve compression. Note an "atypical" activation of proximal extensors during late stance (marked in red) and its variability across patients and depending on the affected side. 
A

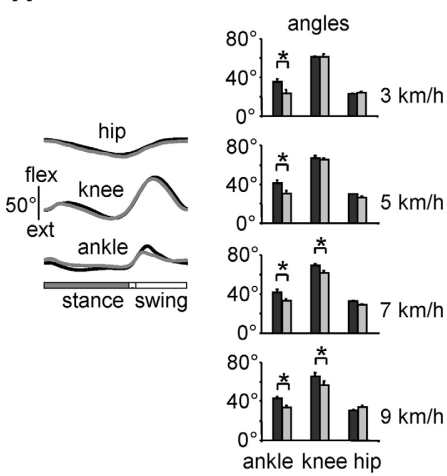

B

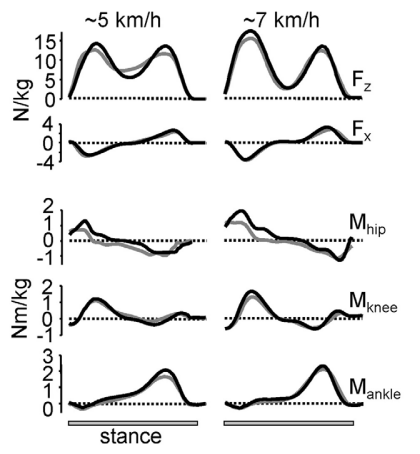

patient 1 (session 2)
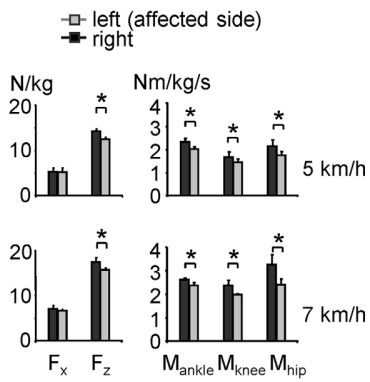

C
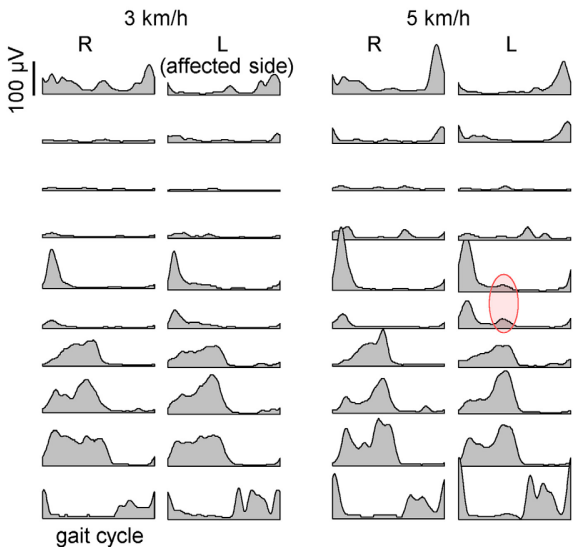

FIGURE 3 | Motor patterns in a patient with sciatic nerve compression [the same patient as in Figure 2A but recorded 1 year later (session 2)]. (A) ensemble-averaged (across 12 consecutive steps) joint angular displacements (left panel, mean $\pm S D$ ) and amplitudes of angular joint motion (right panel) during walking at $3 \mathrm{~km} / \mathrm{h}$. Asterisks denote significant differences. Note significantly smaller distal joint oscillations on the affected (left) side. (B) ensemble-averaged ( $n=5$ steps) vertical $\left(F_{z}\right)$ and horizontal anterior-posterior $\left(F_{x}\right)$ ground reaction forces, and ankle, knee and hip joint moments of force normalized to the patient's weight during overground walking at $\sim 5$ and $7 \mathrm{~km} / \mathrm{h}$ in session 2 (left panels). The patterns are plotted
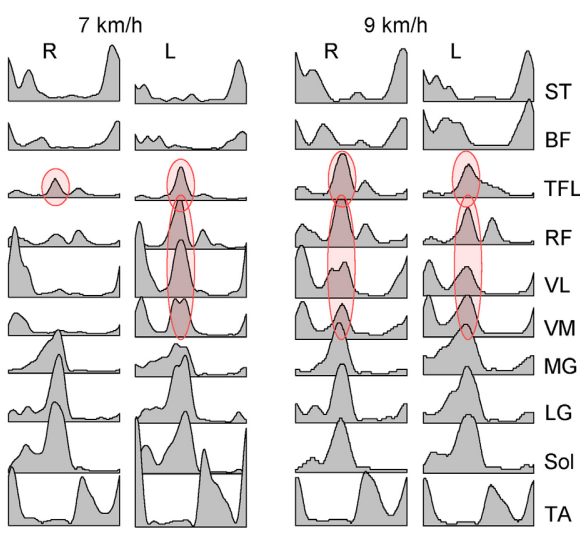

vs. normalized stance. On the right—peak-to-peak amplitudes. (C) Ensemble-averaged bilateral EMG activity of leg muscles during walking on a treadmill at different speeds. In session 1 (Figure 2A), the patient could walk only at speeds up to $5 \mathrm{~km} / \mathrm{h}$ due to plantarflexor weakness, while in session 2 we recorded walking in a wide range of speeds $(3-9 \mathrm{~km} / \mathrm{h})$. Adapted from Ivanenko et al. (2013a). Note a prominent burst of activity (marked in red) in the proximal extensors during late stance on the affected (left) side at low speeds in the session 1 (Figure 2A) and only at higher speeds $(>5 \mathrm{~km} / \mathrm{h})$ in session 2. Furthermore, at $9 \mathrm{~km} / \mathrm{h}$ the "atypical" burst of activity was present in both legs, as in healthy subjects (see Figure 1A). the ankle joint motion (Figure 3A). In the knee joint, angular motion asymmetry was significant only at higher speeds ( 7 and $9 \mathrm{~km} / \mathrm{h}$ ). When the plantarflexor strength and/or reflexes were compromised in one leg, the primary compensatory mechanism was an increase in activity of proximal extensor muscles (VL, RF, VM, TFL evidenced by red circles in Figure 2) during late stance. This compensatory effect was observed at all recorded walking speeds in patient 1 in session 1 (Figure 2A) but only at the higher speeds $(>5 \mathrm{~km} / \mathrm{h}$ ) in session 2 (Figure $3 \mathrm{C}$ ) which required greater propulsion forces. This suggests a possible link to the extent of the weakness in the ankle extensors.

Healthy volunteers typically do not show activity in the proximal extensor muscles during late stance in normal walking (Nilsson et al., 1985; Winter, 1989; Prilutsky and Gregor, 2001; Nene et al., 2004; Cappellini et al., 2006). However, the atypical proximal burst was present in all healthy subjects at high, nonpreferred walking speeds (Figure 1A, right panel). The proximal muscles involved (RF, VM, VL, SART, Gmed, TFL) were activated synchronously with the distal extensors (MG, LG, SOL) as was observed for the patient. At the higher speeds, the muscle gain, or force produced for a given level of activation, may be lower due to the muscle force-velocity relationship (Neptune and Sasaki, 2005), so the force produced by ankle extensors alone may be insufficient to initiate lift-off or to provide appropriate limb stiffness. The proximal activation may be recruited to compensate by supplying additional extensor torque and stiffness.

Similarities can also be noted between this type of adaptation and unilateral acute pathologies such as hemiplegia (Knutsson and Richards, 1979), below-knee amputation (Winter and Sienko, 1988) or unilateral ischemic block of distal leg muscles in healthy subjects (Dickey and Winter, 1992). Co-activation of distal and proximal extensors during stance in each leg was also observed in the clinically incomplete spinal cord injury individuals during weight-bearing treadmill stepping (Beres-Jones and Harkema, 
2004, see also below) and in healthy adults during walking on a slippery surface (Cappellini et al., 2010). Such similarity indicates that this kind of adaptation seems not to depend on the location of the lesion of the neuronal system but may be related to the activation of existing basic activation patterns or muscle synergies.

Nevertheless, despite a potential broad-spectrum functional significance of this compensatory response to plantarflexor weakness, its biomechanical nature remains puzzling. One would perhaps expect the cooperation of distal and proximal extensors during knee-flexed locomotion, e.g., as it happens during digitigrade gait requiring a significant positive (anti-gravity) knee torque during stance. However, in our case (Figure 3B) there was no generation of positive knee moments of force in response to extra activation of knee extensors, likely reflecting dynamic coupling between body segments (Zajac et al., 2003). Moreover, biomechanical simulations of the compensatory strategies in response to muscle weakness do not seem to predict the appearance of "atypical" burst of activity in the proximal extensors (Goldberg and Neptune, 2007). Perhaps a complete consideration of all factors affecting gait optimization is necessary, including a $3 \mathrm{D}$ rather than $2 \mathrm{D}$ musculoskeletal gait model (e.g., Gmed, TFL, and SART may generate a noticeable trunk torsion or lateral force component, Dostal et al., 1986) and taking into account the mechanisms that regulate leg stiffness during walking (Fiolkowski et al., 2005).

Whatever the exact biomechanical reasons for the observed phenomenon (Figure 3), it is important to emphasize that the timing of the reaction in the proximal muscles corresponded to the timing of the calf muscle activation. This supports the idea of a temporal architecture of the locomotor program linked to specific kinematic events (Ivanenko et al., 2005, 2006; Giszter et al., 2007; McGowan et al., 2010) or critical points in the processing of sensory information (Saltiel and Rossignol, 2004) in the gait cycle.

\section{TRANSTIBIAL AMPUTEES}

Various efforts have been made to restore the normal EMG patterns in patients, presumably by reactivating the CPG (central pattern generator) circuitry or more directly by functional electrical stimulation (FES) (Thrasher et al., 2006; Solopova et al., 2011) or when implementing myoelectric control of powered limb prostheses in amputees (Au et al., 2008; Huang et al., 2011). For instance, transtibial amputees can learn to volitionally activate residual leg muscles (Au et al., 2008; Ha et al., 2011; Hargrove et al., 2011) that can be used for movement intent recognition in the myoelectric control of powered limb prostheses. However, such strategies and their underlying hypotheses are often based on the assumption that the motor patterns are relatively invariant across different walking conditions, for instance, when using FES for gait rehabilitation (Thrasher et al., 2006). Below we consider a reorganization of EMG activity in transtibial amputees.

Below-knee amputation represents a severe damage of the neuromuscular apparatus of the leg and impaired sensory feedback. As a result, the EMG activity in amputees may be compromised by these factors. Indeed, below-knee amputation may result in the EMG patterns different from those in healthy subjects.
For instance, co-activation of distal and proximal extensors during stance, similar to that described in the previous section (Figures 2, 3C), was also observed in below-knee amputees (Winter and Sienko, 1988).

Of particular interest is residual lower leg muscle activation following such an amputation. For instance, if the CPG output were relatively fixed (e.g., providing an alternating activity of flexors and extensors, Zehr, 2005) one would not expect major changes in residual muscle activation profiles. Figure 4 illustrates the results of a recent study on multi-muscle EMG activity in both proximal and residual leg muscles during walking in transtibial amputees (Huang and Ferris, 2012). In the upper leg muscles, the data showed that amputee subjects had greater inter-subject variability in their biceps femoris and gluteus medius muscle activation profiles compared to control subjects during walking, as well as a different BF activation profile shape (Figure 4, right panels). Amputee subjects also demonstrated reliable muscle recruitment signals from residual lower leg muscles recorded within the prosthetic socket during walking. However, the grouping of muscles activated together differed from that in controls (see, for instance, "atypical" co-activation of Gmed, BF and MG in the A02 subject or TA, BF and Gmed in the A10 subject). Overall, muscle activation profile variability was higher for amputee subjects than for control subjects (Huang and Ferris, 2012). Nevertheless, it is interesting to note that muscle recruitment signals in amputees tended to be locked to particular phases of the gait cycle (Figure 4).

\section{LOCOMOTOR PATTERNS IN CENTRAL LESIONS}

Contrary to impairments in the peripheral sensory feedback or neuromuscular apparatus, nervous system lesions may essentially affect central controllers and thus provide some insights into the spatiotemporal organization of neural circuitry. In particular, if muscle modules are indeed mechanisms by which task level biomechanical goals are implemented, one would expect that impairments to the neural control of such modules would directly result in impaired biomechanical outputs (Cheung et al., 2009; Ivanenko et al., 2009; Clark et al., 2010). In addition, lesion at different levels of the neuraxis could differentially affect locomotor control. Adaptation of gait after cortical, subcortical or spinal cord damage might thus represent the experimental field in which one might test such hypotheses and the therapeutic relevance of different interventions.

\section{STROKE PATIENTS}

Post-stroke locomotor impairments are often associated with abnormal spatiotemporal patterns of muscle coordination (Knutsson and Richards, 1979; De Quervain et al., 1996; Mulroy et al., 2003; Den Otter et al., 2007). Furthermore, impaired locomotor coordination in post-stroke may be accompanied by fewer modules (Clark et al., 2010; Safavynia et al., 2011), though in a recent study Gizzi et al. (2011) have argued that impulses of activation rather than muscle synergies are preserved in the locomotion of subacute stroke patients (Figure 5). The discrepancies could be accounted for by a different set of recorded muscles or different populations of patients. The authors of both studies hypothesized that identification of motor modules may lead 


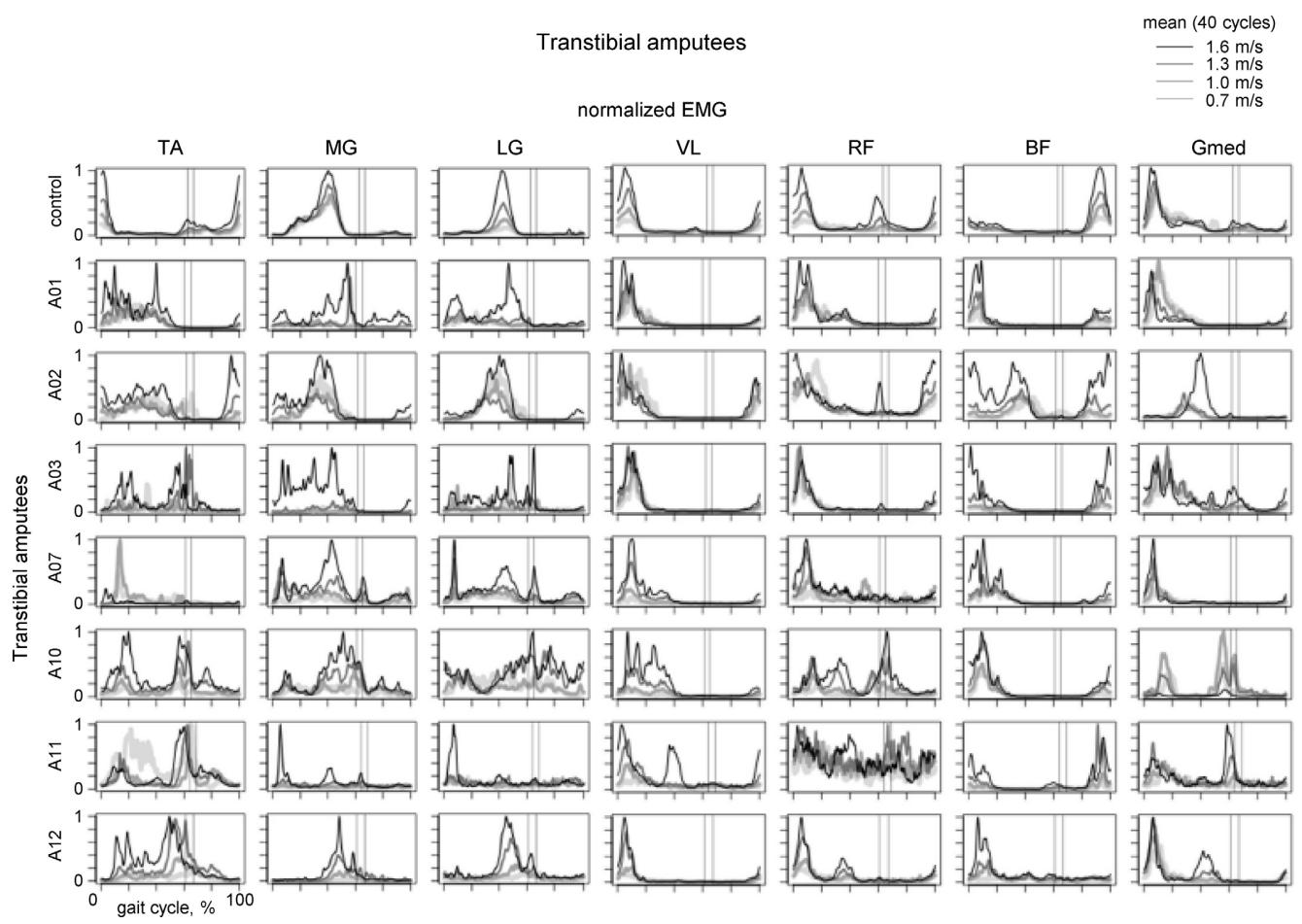

FIGURE 4 | Ensemble-averaged EMG activity of leg muscles during walking at different speeds in the control group (upper panels) and seven amputee subjects. Adapted from
Huang and Ferris (2012) with permission of the authors. Vertical lines show average toe-off events for the fastest and slowest walking speeds. to new insight into how nervous system injury may alter the organization of motor modules and their biomechanical outputs. Furthermore, entraining appropriate motor modules can be of a major importance for neurorehabilitation of gait in these patients since many of them develop an abnormal stereotype of movement during walking, which is difficult to correct.

A similar conclusion has been reached in recent studies on the upper limb control (Cheung et al., 2009, 2012). All patients studied suffered from a mostly unilateral cortical and subcortical lesion resulting from either an ischemic or a hemorrhagic stroke. The robustness of muscle synergies observed in that study supports the notion that descending cortical signals represent neural drives that select, activate, and flexibly combine muscle synergies specified by networks in the spinal cord and/or brainstem and suggest an approach to stroke rehabilitation by focusing on those synergies with altered activations after stroke. Nevertheless, despite higher variability in muscle activation patterns, all these studies suggest a modular organization of muscle coordination underlying motor control in both healthy and post-stroke subjects.

In fact, one of the effective approaches to gait rehabilitation after stroke consists in using step synchronized FES of leg muscles (Yan et al., 2005; Tong et al., 2006; Ferrante et al., 2008). FES has been shown to be an effective tool for muscle strength augmentation, increase in the range of motion in joints and improvements in walking in neurological patients (Popovic et al., 2009). FES is delivered in reference to the timing of natural muscle excitation during movement and it provides additional sensory reinforcement which ultimately improves learning. As a result, the locomotor centers or networks are excited or released from inhibition in phase with their expected activity and thus may be accessible for correction or stimulating effects (Yan et al., 2005; Tong et al., 2006; Popovic et al., 2009; Solopova et al., 2011). Thus, this approach takes advantage of the spatiotemporal architecture of the locomotor program and increases the patient's functional abilities and the effectiveness of rehabilitation.

\section{SPINAL CORD INJURY}

Flexibility and adaptability of locomotor patterns are evident from monitoring and analyzing the spatiotemporal spinal segmental output after spinal cord injury. For instance, in motor incomplete paraplegics who recovered independent control of their limbs, an additional activation burst is present in the lumbosacral enlargement at full loading (Figures 6A,C). The presence of this burst is related to abnormal activation of the quadriceps muscle during this time. Patients can be trained to step with body weight support unassisted, but they use activity patterns in individual muscles that were often different from healthy individuals.

A number of clinical trials have suggested the possible beneficial effects of locomotor training in SCI patients (Edgerton and Roy, 2012). In patients with severe SCI disorders, initial training is performed while being supported by a harness or with their body partially unloaded. As well, assistance of leg movements by the therapist (or robotics) may be required. These patients frequently show EMG patterns different from those of healthy 


\section{Stroke patients}

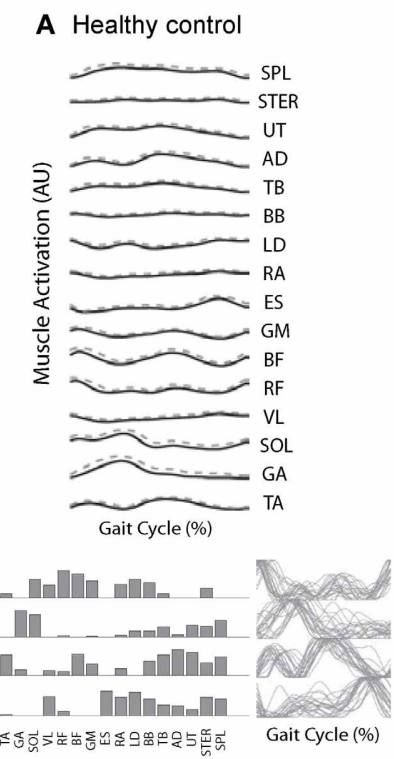

B Patient unaffected side
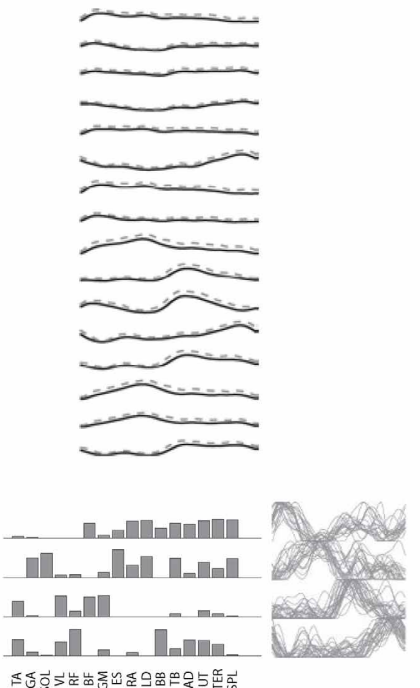

C Patient affected side
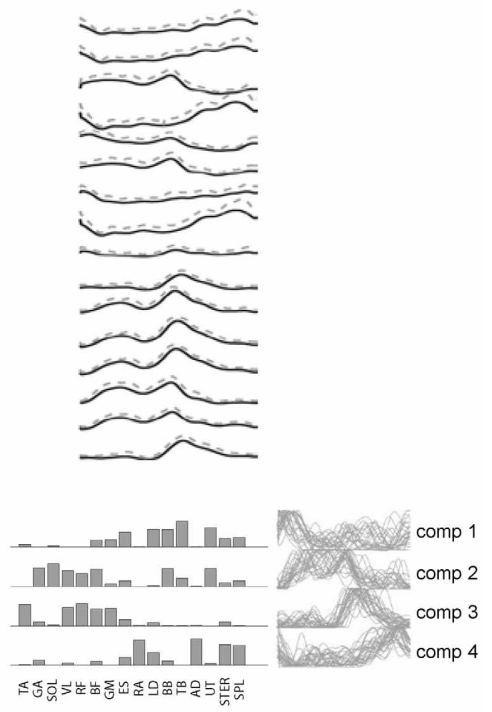

FIGURE 5 | Surface electromyogram (means and SD), motor modules (bottom, left), and activation signals (bottom, right) for a representative healthy control subject (A) and for the unaffected (B) and affected side of a stroke patient. (C) Adapted from Gizzi et al. (2011).

individuals suggesting that human spinal cord can interpret differently loading- or velocity-dependent sensory input during stepping (Beres-Jones and Harkema, 2004). One method used to study such variability involves reconstructing the total output pattern of motoneuron activity of the lumbosacral enlargement of the spinal cord by mapping the recorded EMG waveforms onto the known charts of segmental localization (Ivanenko et al., 2006). Spatiotemporal maps of motoneuron activity are generally different from those of healthy subjects (Figure 6C; Grasso et al., 2004). The legs may also show muscle activity which is not-systematically synchronized with the gait cycle on the most affected side (Figure 6B; marked in red). Although training of stepping in patients with body weight support can be facilitated in a laboratory setting, often new coordinative strategies appear. Training can be utilized in order for patients to relearn foot kinematics of healthy individuals, but the muscle activation patterns used to generate these kinematics differ from that of a healthy group (Pepin et al., 2003; Grasso et al., 2004). While most incomplete paraplegics can recover independent control of leg muscles sufficient to propel the limbs in swing and to support body weight in stance, complete paraplegics, were unable to and they typically used their arms and body to assist the leg movements. SCI patients largely relied on proximal and axial muscles to lift the foot and to project the limb forward.

It is though that spinal lesions may trigger plasticity including modified synaptic strengths, sprouting and anatomical development of new circuits as well as plasticity of unlesioned descending pathways, including both subcortical and cortical motor areas. Stepping may also depend more heavily on cortical (and voluntary) control after severe spinal lesions (Van den Brand et al.,
2012) than it does in healthy subjects, where locomotion may be more automatic. The spinal cord itself does also contribute to the proposed adaptation mechanisms. Indeed, experiments on both animals and SCI patients suggest that the spinal cord is capable of adaptive locomotor plasticity with training after spinal lesion (Hodgson et al., 1994; Belanger et al., 1996; Heng and de Leon, 2007) or after peripheral motor nerve lesions (Bouyer et al., 2001).

Modular pattern generator elements (or burst synergies), nevertheless, tend to be conserved after spinal cord injury (Fox et al., 2013; Giszter and Hart, 2013). Our previous work in SCI patients (Ivanenko et al., 2003) has shown a similar set of temporal components from EMG activity (Figure 6D). In addition, muscles both rostral and caudal to the lesions could be strongly weighted on a given component. However, some patients do exhibit a smaller number of basic components during walking (Ivanenko et al., 2003; Hayes et al., 2011; Fox et al., 2013) although these results may be dependent on the number and selection of muscles recorded during stepping. Nevertheless, similar activation timings in SCI patients (Figure 6D) may be ultimately related to the global kinematic goal (a motor equivalent solution; Grasso et al., 2004) or the necessity to apply forces at particular phases of the gait cycle (Lacquaniti et al., 2012). These data highlight the importance of understanding the modular structure of motor behaviors to best provide principled therapies after central nervous system lesions (Giszter and Hart, 2013).

\section{CONCLUDING REMARKS}

Taken together, the data support the idea of plasticity and distributed networks for controlling human locomotion (Scivoletto 


\section{SCl patients}
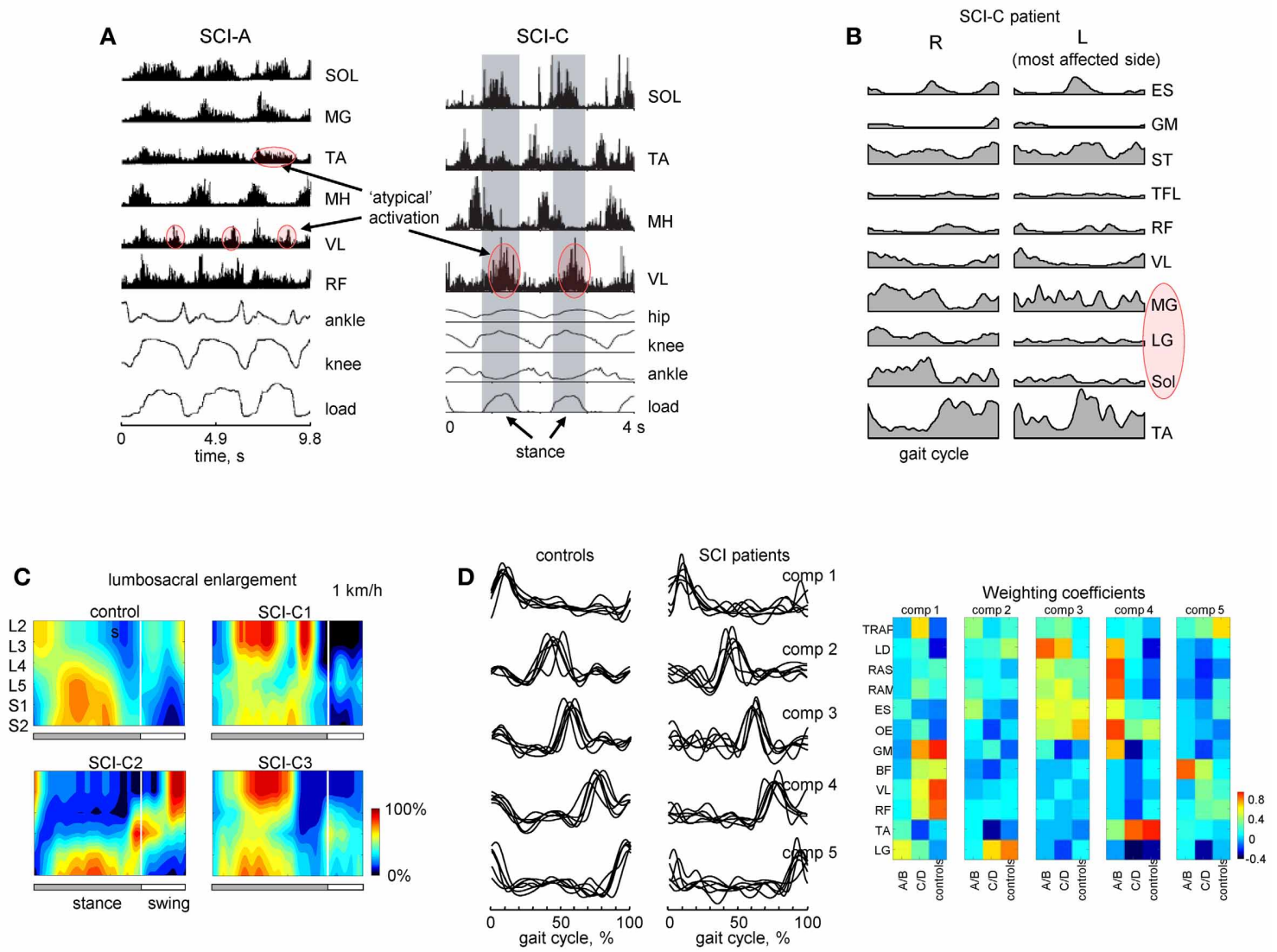

FIGURE 6 | Motor patterns in SCI patients. (A) An example of weight-bearing stepping in a clinically complete (at $0.22 \mathrm{~m} / \mathrm{s}$, left panel) and incomplete (at $0.89 \mathrm{~m} / \mathrm{s}$, right panel) $\mathrm{SCl}$ individuals [modified from Beres-Jones and Harkema (2004) and Maegele et al. (2002) with permission of the authors]. The stance phase in the right panel is evidenced by the elevation in the ground reaction force trace and indicated by the shaded region. $\mathrm{MH}$, medial hamstring; load, vertical ground reaction force. (B) Ensemble-averaged (across 5 strides) EMG patterns in the $\mathrm{SCl}-\mathrm{C}$ patient during walking at a natural speed $(\sim 3.1 \mathrm{~km} / \mathrm{h})$. Note variable and weaker muscle activity on the most affected side (marked in red). (C) Examples of spatiotemporal patterns of $\alpha$-motorneuron activity in the lumbosacral enlargement in controls and three $\mathrm{SCl}-\mathrm{C}$ patients during walking on a treadmill at $1 \mathrm{~km} / \mathrm{h}$. Output pattern for each segment was reconstructed by mapping the recorded EMG waveforms (normalized method, see Ivanenko et al., 2006) onto the known charts of segmental localization. White vertical lines denote stance-to-swing transition time. (D) Time course of the temporal components in controls and patients for stepping at $2 \mathrm{~km} / \mathrm{h}, 0-75 \%$ body weight support. The components extracted by factor analysis from individual subjects. Right panel illustrates weighting coefficients of the temporal components in individual activity patterns of 12 muscles for all groups of subjects in a color coded scale. Adapted from Ivanenko et al. (2003). Note similar basic EMG components in controls and patients as opposed to quite different EMG patterns and weighting coefficients. et al., 2007). Tens of muscles participate in the control of limb and body movements during locomotion, and redundancy in the neuromuscular system is an essential element of gait adaptability (Winter, 1989; Cai et al., 2006; Noble and Prentice, 2006; Ivanenko et al., 2009; Molinari, 2009; Duysens et al., 2013). Indeed, experimental studies performed on individuals with wellidentified pathologies have demonstrated distinct adaptations. Due to muscle redundancy, various neuromotor strategies may exist to compensate for decreased muscle strength and joint stiffness (Grasso et al., 2004; Goldberg and Neptune, 2007; Ivanenko et al., 2009; Gordon et al., 2013).

A modular motor organization may be needed to solve the degrees of freedom problem in biological motor control (Giszter et al., 2010). Nevertheless, there are still many open questions related to the choice of appropriate modules, their task dependence, influence of sensory input and adaptation to the malfunctioning of neural networks in the case of different gait pathologies. While many studies succeeded in a decomposition of motor patterns into a few "motor modules," nevertheless, the way in which the central nervous system combines them together, how and where the weighting coefficients are encoded are not understood. Often it is difficult to distinguish what primarily comes from pathology and what comes from compensatory mechanisms. We suggest that many adaptive features in various neurological disorders are likely compensatory, including modified EMG patterns during walking. In view of task dependence of muscle synergies, it would be interesting also to compare them in different behaviors and examine whether plasticity in muscle patterns originates from sharing these common modules or by creating new muscle synergies. 
An impulsive (burst-like) controller made of a lowdimensional set of time-delayed excitation pulses has been also thoroughly considered in a simulation study from the biomechanical viewpoint (Sartori et al., 2013). In particular, simulated gait motions based on a few modular activation patterns were successfully produced (see also Neptune et al., 2009; Allen and Neptune, 2012; Allen et al., 2013). Once calibrated, the musculoskeletal model could work in open-loop, approximating joint moments over multiple degrees of freedom using only the recorded kinematics and the internal impulsive controller. The accuracy of estimation of the joint torques was comparable when using the low-dimensional activation signals (Sartori et al., 2013). This approach has substantial implications for the design of human machine interfaces for prosthetic and orthotic devices.

\section{REFERENCES}

Allen, J. L., and Neptune, R. R. (2012). Three-dimensional modular control of human walking. J. Biomech. 45, 2157-2163. doi: 10.1016/j.jbiomech.2012.05.037

Allen, J. L., Kautz, S. A., and Neptune, R. R. (2013). The influence of merged muscle excitation modules on post-stroke hemiparetic walking performance. Clin. Biomech. (Bristol, Avon). 28, 697-704. doi: 10.1016/j.clinbiomech.2013.06.003

$\mathrm{Au}$, S., Berniker, M., and Herr, H. (2008). Powered ankle-foot prosthesis to assist levelground and stair-descent gaits. Neural. Netw. 21, 654-666. doi: 10.1016/j.neunet.2008.03.006

Belanger, M., Drew, T., Provencher, J., and Rossignol, S. (1996). A comparison of treadmill locomotion in adult cats before and after spinal transection. J. Neurophysiol. 76, 471-491.

Beres-Jones, J. A., and Harkema, S. J. (2004). The human spinal cord interprets velocity-dependent afferent input during stepping. Brain 127, 2232-2246. doi: 10.1093/brain/awh252

Bizzi, E., and Cheung, V. C. (2013). The neural origin of muscle synergies. Front. Comput. Neurosci. 7:51. doi: 10.3389/fncom.2013.00051

Bouyer, L. J., Whelan, P. J., Pearson, K. G., and Rossignol, S. (2001). Adaptive locomotor plasticity in chronic spinal cats after ankle extensors neurectomy. J. Neurosci. 21, 3531-3541.

Bramble, D. M., and Lieberman, D. E. (2004). Endurance running and the evolution of Homo. Nature 432, 345-352. doi: 10.1038/nature03052

Brunner, R., and Romkes, J. (2008). Abnormal EMG muscle activity during gait in patients without neurological disorders.
Gait Posture 27, 399-407. doi: 10.1016/j.gaitpost.2007.05.009

Cai, L. L., Courtine, G., Fong, A. J., Burdick, J. W., Roy, R. R., and Edgerton, V. R. (2006). Plasticity of functional connectivity in the adult spinal cord. Philos. Trans. R. Soc. Lond. B Biol. Sci. 361, 1635-1646. doi: 10.1098/rstb.2006. 1884

Cao, Y., C'Olhaberriague, L., Vikingstad, E. M., Levine, S. R., and Welch, K. M. A. (1998). Pilot study of functional MRI to assess cerebral activation of motor function after post-stroke hemiparesis. Stroke 29, 112-122. doi: 10.1161/01.STR.29.1.112

Cappellini, G., Ivanenko, Y. P., Dominici, N., Poppele, R. E., and Lacquaniti, F. (2010). Motor patterns during walking on a slippery walkway. J. Neurophysiol. 103, 746-760. doi: 10.1152/jn.00499. 2009

Cappellini, G., Ivanenko, Y. P., Poppele, R. E., and Lacquaniti, F. (2006). Motor patterns in human walking and running. J. Neurophysiol. 95, 3426-3437. doi: 10.1152/jn.00081.2006

Cheung, V. C., Piron, L., Agostini, M. Silvoni, S., Turolla, A., and Bizzi, E. (2009). Stability of muscle synergies for voluntary actions after cortical stroke in humans. Proc. Natl. Acad. Sci. U.S.A. 106, 19563-19568. doi: 10.1073/pnas.0910114106

Cheung, V. C., Turolla, A., Agostini, M., Silvoni, S., Bennis, C., Kasi, P., et al. (2012). Muscle synergy patterns as physiological markers of motor cortical damage. Proc. Natl. Acad. Sci. U.S.A. 109, 14652-14656. doi: 10.1073/pnas.1212056109

Chvatal, S. A., and Ting, L. H. (2012). Voluntary and reactive recruitment of locomotor muscle synergies during perturbed walking.

Uncovering a common underlying neural framework for the modular control of human locomotion and its development represent an interesting avenue for the future work. Motor primitives may reflect in a way how the nervous system develops, by building up or modifying modules as it matures. Some functional units are likely inborn, others may develop later or be dependent on individual body size/proportions or experience (Dominici et al., 2011). Such investigations may have important implications related to the construction of gait rehabilitation technology.

\section{ACKNOWLEDGMENTS}

Supported by the Italian Ministry of Health, Italian University Ministry (PRIN project), Italian Space Agency (DCMC and CRUSOE grants) and European Union FP7-ICT program (MINDWALKER grant \# 247959 and AMARSi grant \#248311).

J. Neurosci. 32, 12237-12250. doi: 10.1523/JNEUROSCI.6344-11.2012

Clark, D. J., Ting, L. H., Zajac, F. E., Neptune, R. R., and Kautz, S. A. (2010). Merging of healthy motor modules predicts reduced locomotor performance and muscle coordination complexity post-stroke. J. Neurophysiol. 103, 844-857. doi: 10.1152/jn.00825. 2009

Courtine, G., Papaxanthis, C., and Schieppati, M. (2006). Coordinated modulation of locomotor muscle synergies constructs straight-ahead and curvilinear walking in humans. Exp. Brain Res. 170, 320-335. doi: 10.1007/s00221-005-0215-7

d'Avella, A., and Bizzi, E. (2005) Shared and specific muscle synergies in natural motor behaviors. Proc. Natl. Acad. Sci. U.S.A. 102, 3076-3081. doi: 10.1073/pnas.050 0199102

Davis, B. L., and Vaughan, C. L. (1993). Phasic behavior of EMG signals during gait: use of multivariate statistics. J. EMG Kinesiol. 3, 51-60. doi: 10.1016/1050-6411(93)90023-P

Den Otter, A. R., Geurts, A. C. Mulder, T., and Duysens, J. (2007). Abnormalities in the temporal patterning of lower extremity muscle activity in hemiparetic gait. Gait Posture 25, 342-352. doi: 10.1016/j.gaitpost.2006.04.007

De Quervain, I. A., Simon, S. R. Leurgans, S., Pease, W. S., and McAllister, D. (1996). Gait pattern in the early recovery period after stroke. J. Bone Joint Surg. Am. 78, 1506-1514.

Dickey, J. P., and Winter, D. A. (1992). Adaptations in gait resulting from unilateral ischaemic block of the leg. Clin. Biomech. 7, 215-225. doi: 10.1016/S0268-0033(92)90004-N

Dominici, N., Ivanenko, Y. P., Cappellini, G., d'Avella, A.,
Mondì, V., Cicchese, M., et al. (2011). Locomotor primitives in newborn babies and their development. Science 334, 997-999. doi: 10.1126/science.1210617

Dostal, W. F., Soderberg, G. L., and Andrews, J. G. (1986). Actions of hip muscles. Phys. Ther. 66, 351-361.

Duysens, J., De Groote, F., and Jonkers, I. (2013). The flexion synergy, mother of all synergies and father of new models of gait. Front. Comput. Neurosci. 7:14. doi: 10.3389/fncom.2013.00014

Edgerton, V. R., and Roy, R. R. (2012). A new age for rehabilitation. Eur. J. Phys. Rehabil. Med. 48, 99-109.

Ferrante, S., Pedrocchi, A., Ferrigno, G., and Molteni, F. (2008). Cycling induced by functional electrical stimulation improves the muscular strength and the motor control of individuals with post-acute stroke. Eur. J. Phys. Rehabil. Med. 44, 159-167.

Fiolkowski, P., Bishop, M., Brunt, D., and Williams, B. (2005). Plantar feedback contributes to the regulation of leg stiffness. Clin. Biomech. 20, 952-958. doi: 10.1016/j.clinbiomech.2005.03.013

Forssberg, H. (1985). Ontogeny of human locomotor control. I. Infant stepping, supported locomotion and transition to independent locomotion. Exp. Brain Res. 57, 480-493. doi: 10.1007/BF00237835

Fox, E. J., Tester, N. J., Kautz, S. A., Howland, D. R., Clark, D. J., Garvan, C., et al. (2013). Modular control of varied locomotor tasks in children with incomplete spinal cord injuries. J. Neurophysiol. doi: 10.1152/jn.00676.2012. [Epub ahead of print].

Giszter, S., Patil, V., and Hart, C. (2007). Primitives, premotor drives, and pattern generation: a combined computational and 
neuroethological perspective. Prog. Brain Res. 165, 323-346. doi: 10.1016/S0079-6123(06)65020-6

Giszter, S. F., and Hart, C. B. (2013). Motor primitives and synergies in the spinal cord and after injurythe current state of play. Ann. N.Y. Acad. Sci. 1279, 114-126. doi: 10.1111/nyas. 12065

Giszter, S. F., Hart, C. B., and Silfies, S. P. (2010). Spinal cord modularity: evolution, development, and optimization and the possible relevance to low back pain in man. Exp. Brain Res. 200, 283-306. doi: 10.1007/s00221-009-2016-x

Gizzi, L., Nielsen, J. F., Felici, F., Ivanenko, Y. P., and Farina, D. (2011). Impulses of activation but not motor modules are preserved in the locomotion of subacute stroke patients. J. Neurophysiol. 106, 202-210. doi: 10.1152/jn.00727. 2010

Goldberg, E. J., and Neptune, R. R. (2007). Compensatory strategies during normal walking in response to muscle weakness and increased hip joint stiffness. Gait Posture 25, 360-367. doi: 10.1016/j.gaitpost.2006.04.009

Gordon, K. E., Kinnaird, C. R., and Ferris, D. P. (2013). Locomotor adaptation to a soleus EMGcontrolled antagonistic exoskeleton. J. Neurophysiol. 109, 1804-1814. doi: 10.1152/jn.01128.2011

Grasso, R., Bianchi, L., and Lacquaniti, F. (1998). Motor patterns for human gait: backward versus forward locomotion. J. Neurophysiol. 80, 1868-1885.

Grasso, R., Ivanenko, Y. P., Zago, M., Molinari, M., Scivoletto, G., Castellano, V., et al. (2004). Distributed plasticity of locomotor pattern generators in spinal cord injured patients. Brain 127, 1019-1034. doi: 10.1093/brain/ awh 115

Ha, K. H., Varol, H. A., and Goldfarb, M. (2011). Volitional control of a prosthetic knee using surface electromyography. IEEE Trans. Biomed. Eng. 58, 144-151. doi: 10.1109/TBME.2010.2070840

Hagiwara, Y., Hatori, M., Kokubun, S., and Miyasaka, Y. (2003). Gait characteristics of sciatic nerve palsy-a report of four cases. Ups. J. Med. Sci. 108, 221-227. doi: 10.3109/20001967-116

Hargrove, L. J., Simon, A. M., Lipschutz, R. D., Finucane, S. B., and Kuiken, T. A. (2011). Realtime myoelectric control of knee and ankle motions for transfemoral amputees. JAMA 305, 1542-1544. doi: 10.1001/jama.2011.465
Hayes, H. B., Van Hiel, L. R., Chvatal, S. A., Ting, L. H., Tansey, K. E., and Trumbower, R. D. (2011). Modularity of muscle activity during robot-assisted locomotion in persons within complete spinal cord injury. Abstract presented at Annual Meeting 2011, Society for Neuroscience (Washington, DC).

Heng, C., and de Leon, R. D. (2007). The rodent lumbar spinal cord learns to correct errors in hindlimb coordination caused by viscous force perturbations during stepping. J. Neurosci. 27, 8558-8562. doi: 10.1523/JNEUROSCI.1635-07. 2007

Hodgson, J. A., Roy, R. R., de Leon, R., Dobkin, B., and Edgerton, V. R. (1994). Can the mammalian lumbar spinal cord learn amotor task. Med. Sci. Sports Exerc. 26, 1491-1497.

Huang, H., Zhang, F., Hargrove, L. J., Dou, Z., Rogers, D. R., and Englehart, K. B. (2011). Continuous locomotion-mode identification for prosthetic legs based on neuromuscular-mechanical fusion. IEEE Trans. Biomed. Eng. 58, 2867-2875. doi: 10.1109/TBME. 2011.2161671

Huang, S., and Ferris, D. P. (2012). Muscle activation patterns during walking from transtibial amputees recorded within the residual limbprosthetic interface. J. Neuroeng. Rehabil. 9, 55. doi: 10.1186/17430003-9-55

Ivanenko, Y. P., Cappellini, G., Dominici, N., Poppele, R. E., and Lacquaniti, F. (2005). Coordination of locomotion with voluntary movements in humans. J. Neurosci. 25, 7238-7253. doi: 10.1523/ JNEUROSCI.1327-05.2005

Ivanenko, Y. P., Cappellini, G., Poppele, R. E., and Lacquaniti, F. (2008). Spatiotemporal organization of alpha-motoneuron activity in the human spinal cord during different gaits and gait transitions. Eur. J. Neurosci. 27, 3351-3368. doi: 10.1111/j.1460-9568.2008.06289.X

Ivanenko, Y. P., Cappellini, G., Solopova, I. A., Grishin, A. A., MacLellen, M. J., Poppele, R. E., et al. (2013a). "Plasticity and different solutions to reorganize muscle patterns during gait," in Converging Clinical and Engineering Research on Neurorehabilitation. Series: Biosystems and Biorobotics, Vol. 1, eds J. L. Pons, D. Torricelli, and M. Pajaro (Heidelberg: Springer), 732.

Ivanenko, Y. P., Dominici, N., Cappellini, G., Di Paolo, A., Giannini, C., Poppele, R. E., et al. (2013b). Changes in the spinal segmental motor output for stepping during development from infant to adult. J. Neurosci. 33, 3025-3036a. doi: 10.1523/JNEUROSCI.2722-12.2013

Ivanenko, Y. P., Dominici, N., and Lacquaniti, F. (2007). Development of independent walking in toddlers. Exerc. Sport Sci. Rev. 35, 67-73. doi: 10.1249/JES.0b013e31803eafa8

Ivanenko, Y. P., Grasso, R., Macellari, V., and Lacquaniti, F. (2002). Control of foot trajectory in human locomotion: role of ground contact forces in simulated reduced gravity. J. Neurophysiol. 87, 3070-3089.

Ivanenko, Y. P., Grasso, R., Zago, M., Molinari, M., Scivoletto, G., Castellano, V., et al. (2003). Temporal components of the motor patterns expressed by the human spinal cord reflect foot kinematics. J. Neurophysiol. 90, 3555-3565. doi: 10.1152/jn.00223.2003

Ivanenko, Y. P., Poppele, R. E., and Lacquaniti, F. (2006). Motor control programs and walking. Neuroscientist 12, 339-348. doi: 10.1177/1073858406287987

Ivanenko, Y. P., Poppele, R. E., and Lacquaniti, F. (2009). Distributed neural networks for controlling human locomotion: lessons from normal and SCI subjects. Brain Res. Bull. 78, 13-21. doi: 10.1016/j.brainresbull.2008.03.018

Judge, J. O., Davis, R. B. 3rd., and Ounpuu, S. (1996). Step length reductions in advanced age: the role of ankle and hip kinetics. J. Gerontol. A Biol. Med. Sci. 51, M303-M312.

Knutsson, E., and Richards, C. (1979). Different types of disturbed motor control in gait of hemiparetic patients. Brain 102, 405-430. doi: 10.1093/brain/102.2.405

Lacquaniti, F., Ivanenko, Y. P., d'Avella, A., Zelik, K. E., and Zago, M. (2013). Evolutionary and developmental modules. Front. Comput. Neurosci. 7:61. doi: 10.3389/fncom. 2013.00061

Lacquaniti, F., Ivanenko, Y. P., and Zago, M. (2002). Kinematic control of walking. Arch. Ital. Biol. 140, 263-272.

Lacquaniti, F., Ivanenko, Y. P., and Zago, M. (2012). Patterned control of human locomotion. J. Physiol. 590, 2189-2199. doi: 10.1113/jphysiol.2011.215137

Maegele, M., Muller, S., Wernig, A., Edgerton, V. R., and Harkema, S. J. (2002). Recruitment of spinal motor pools during voluntary movements versus stepping after human spinal cord injury. J. Neurotrauma 19, 1217-1229. doi: $10.1089 / 08977150260338010$
Mazzaro, N., Grey, M. J., Sinkjaer, T., Andersen, J. B., Pareyson, D., and Schieppati, M. (2005). Lack of on-going adaptations in the soleus muscle activity during walking in patients affected by large-fiber neuropathy. J. Neurophysiol. 93, 3075-3085. doi: 10.1152/jn.01071.2004

McGowan, C. P., Neptune, R. R., Clark, D. J., and Kautz, S. A. (2010). Modular control of human walking: adaptations to altered mechanical demands. J. Biomech. 43, 412-419. doi: $\quad$ 10.1016/j.jbiomech.2009. 10.009

Molinari, M. (2009). Plasticity properties of $\mathrm{CPG}$ circuits in humans: impact on gait recovery. Brain Res. Bull. 78, 22-25. doi: 10.1016/j.brainresbull.2008.02.030

Monaco, V., Ghionzoli, A., and Micera, S. (2010). Age-related modifications of muscle synergies and spinal cord activity during locomotion. J. Neurophysiol. 104, 2092-2102. doi: 10.1152/jn.00525.2009

Mulroy, S., Gronley, J., Weiss, W., Newsam, C., and Perry, J. (2003). Use of cluster analysis for gait pattern classification of patients in the early and late recovery phases following stroke. Gait Posture 18, 114-125. doi: 10.1016/S09666362(02)00165-0

Nadeau, S., Gravel, D., Arsenault, A. B., and Bourbonnais, D. (1999). Plantarflexor weakness as a limiting factor of gait speed in stroke subjects and the compensating role of hip flexors. Clin. Biomech. 14, 125-135. doi: 10.1016/S02680033(98)00062-X

Nardone, A., Grasso, M., and Schieppati, M. (2006). Balance control in peripheral neuropathy: are patients equally unstable under static and dynamic conditions. Gait Posture 23, 364-373. doi: 10.1016/j.gaitpost.2005.04.002

Nardone, A., and Schieppati, M. (2004). Group II spindle fibres and afferent control of stance. Clues from diabetic neuropathy. Clin. Neurophysiol. 115, 779-789. doi: 10.1016/j.clinph.2003.11.007

Nardone, A., Tarantola, J., Miscio, G., Pisano, F., Schenone, A., and Schieppati, M. (2000). Loss of large-diameter spindle afferent fibres is not detrimental to the control of body sway during upright stance: evidence from neuropathy. Exp. Brain Res. 135, 155-162. doi: 10.1007/s002210000513

Nelles, G., Spiekermann, G., Jueptner, M., Leonhardt, G., Muller, S., Gerhard, H., et al. (1999). Reorganization of sensory and 
motor system in hemiplegic stroke patients: a positron emission tomography study. Stroke 30, 1510-1516. doi: 10.1161/ 01.STR.30.8.1510

Nene, A., Byrne, C., and Hermens, H. (2004). Is rectus femoris really a part of quadriceps. Assessment of rectus femoris function during gait in able-bodied adults. Gait Posture 20, 1-13. doi: 10.1016/S09666362(03)00074-2

Neptune, R. R., and Sasaki, K. (2005). Ankle plantar flexor force production is an important determinant of the preferred walk-to-run transition speed. J. Exp. Biol. 208, 799-808. doi: 10.1242/jeb. 01435

Neptune, R. R., Clark, D. J., and Kautz, S. A. (2009). Modular control of human walking: a simulation study. J. Biomech. 42, 1282-1287. doi: 10.1016/j.jbiomech.2009.03.009

Nilsson, J., Thorstensson, A., and Halbertsma, J. (1985). Changes in leg movements and muscle activity with speed of locomotion and mode of progression in humans. Acta Physiol. Scand. 123, 457-475. doi: 10.1111/j.17481716.1985.tb07612.x

Noble, J. W., and Prentice, S. D. (2006). Adaptation to unilateral change in lower limb mechanical properties during human walking. Exp. Brain Res. 169, 482-495. doi: 10.1007/s00221-005-0162-3

Pepin, A., Norman, K. E., and Barbeau, H. (2003). Treadmill walking in incomplete spinal-cord-injured subjects. 1. Adaptation to changes in speed. Spinal Cord 41, 257-270. doi: 10.1038/sj.sc.3101452

Popovic, D. B., Sinkaer, T., and Popovic, M. B. (2009). Electrical stimulation as a means for achieving recovery of function in stroke patients. Neurorehabilitation 25, 45-58. doi: 10.3233/NRE-2009-0498

Prentice, S. D., Patla, A. E., and Stacey, D. A. (1998). Simple artificial neural network models can generate basic muscle activity patterns for human locomotion at different speeds. Exp. Brain Res. 123,
474-480. doi: 10.1007/s00221005 0591

Prilutsky, B. I., and Gregor, R. J. (2001). Swing- and support-related muscle actions differentially trigger human walk-run and runwalk transitions. J. Exp. Biol. 204, 2277-2287.

Safavynia, S. A., Torres-Oviedo, G., and Ting, L. H. (2011). Muscle synergies: implications for clinical evaluation and rehabilitation of movement. Top Spinal Cord Inj. Rehabil. 17, 16-24. doi: 10.1310/ scil701-16

Saltiel, P., and Rossignol, S. (2004). Critical points in the forelimb fictive locomotor cycle and motor coordination: evidence from the effects of tonic proprioceptive perturbations in the cat. J. Neurophysiol. 92, 1329-1341. doi: 10.1152/jn.00563.2003

Sartori, M., Gizzi, L., Lloyd, D. G. and Farina, D. (2013). A musculoskeletal model of human locomotion driven by a low dimensional set of impulsive excitation primitives. Front. Comput. Neurosci. 7:79. doi: 10.3389/fncom.2013.00079

Scivoletto, G., Ivanenko, Y. P., Morganti, B., Grasso, R., Zago, M., Lacquaniti, F., et al. (2007) Plasticity of spinal centers in spinal cord injury patients: new concepts for gait evaluation and training. Neurorehabil. Neural Repair 21, 358-365. doi: 10.1177/154596830 6295561

Solopova, I. A., Tihonova, D. Y., Grishin, A. A., and Ivanenko, Y. P. (2011). Assisted leg displacements and progressive loading by a tilt table combined with FES promote gait recovery in acute stroke. Neurorehabilitation 29, 67-77.

Teulier, C., Sansom, J. K., Muraszko, K., and Ulrich, B. D. (2012). Longitudinal changes in muscle activity during infants' treadmill stepping. J. Neurophysiol. 108, 853-862. doi: 10.1152/jn.01037. 2011

Thrasher, T. A., Flett, H. M., and Popovic, M. R. (2006). Gait training regimen for incomplete spinal cord injury using functional electrical stimulation. Spinal Cord 44, 357-361. doi: 10.1038/sj. sc. 3101864

Tong, R. K., Ng, M. F., Li, L. S. and So, E. F. (2006). Gait training of patients after stroke using an electromechanical gait trainer combined with simultaneous functional electrical stimulation. Phys. Ther. 86, 1282-1294. doi: 10.2522/ ptj.20050183

Tresch, M. C., Cheung, V. C., and d'Avella, A. (2006). Matrix factorization algorithms for the identification of muscle synergies: evaluation on simulated and experimental data sets. J. Neurophysiol. 95, 2199-2212. doi: 10.1152/jn.00222. 2005

Tresch, M. C., and Jarc, A. (2009). The case for and against muscle synergies. Curr. Opin. Neurobiol. 19, 601-607. doi: 10.1016/j.conb.2009. 09.002

Van den Brand, R., Heutschi, J., Barraud, Q., DiGiovanna, J., Bartholdi, K., Huerlimann, M. et al. (2012). Restoring voluntary control of locomotion after paralyzing spinal cord injury. Science 336, 1182-1185. doi: 10.1126/science. 1217416

Winter, D. A. (1989). Biomechanics of normal and pathological gait: implications for understanding human locomotor control. J. Mot. Behav. 21, 337-355. doi: 10.1080/00222895.1989.10735488

Winter, D. A., Patla, A. E., Frank, J. S., and Walt, S. E. (1990) Biomechanical walking pattern changes in the fit and healthy elderly. Phys. Ther. 70, 340-347.

Winter, D. A., and Sienko, S. E (1988). Biomechanics of belowknee amputee gait. J. Biomech. 21, 361-367. doi: 10.1016/0021-9290 (88)90142-X

Winter, D. A., and Yack, H. J. (1987). EMG profiles during normal human walking: stride-to-stride and inter-subject variability. Electroencephalogr. Clin. Neurophysiol. 67, 402-411. doi: 10.1016/0013-4694(87)90003-4
Yan, T., Hui-Chan, C. W. Y., and Li, L. S. W. (2005). Functional electrical stimulation improves motor recovery of the lower extremity and walking ability of subjects with first acute stroke. Stroke 36, 80-85. doi: 10.1161/01.STR.0000149623. 24906.63

Zajac, F. E., Neptune, R. R., and Kautz, S. A. (2003). Biomechanics and muscle coordination of human walking Part II: lessons from dynamical simulations and clinical implications. Gait Posture 17, 1-17. doi: 10.1016/S0966-6362(02) 00069-3

Zehr, E. P. (2005). Neural control of rhythmic human movement: the common core hypothesis. Exerc. Sport Sci. Rev. 33, 54-60.

Conflict of Interest Statement: The authors declare that the research was conducted in the absence of any commercial or financial relationships that could be construed as a potential conflict of interest.

Received: 30 November 2012; accepted: 16 August 2013; published online: 10 September 2013.

Citation: Ivanenko YP, Cappellini G, Solopova IA, Grishin AA, MacLellan MJ, Poppele RE and Lacquaniti F (2013) Plasticity and modular control of locomotor patterns in neurological disorders with motor deficits. Front. Comput. Neurosci. 7:123. doi: 10.3389/fncom. 2013.00123

This article was submitted to the journal Frontiers in Computational Neuroscience.

Copyright (C) 2013 Ivanenko, Cappellini, Solopova, Grishin, MacLellan, Poppele and Lacquaniti. This is an open-access article distributed under the terms of the Creative Commons Attribution License (CC BY). The use, distribution or reproduction in other forums is permitted, provided the original author(s) or licensor are credited and that the original publication in this journal is cited, in accordance with accepted academic practice. No use, distribution or reproduction is permitted which does not comply with these terms. 